\title{
Report of the Phenotype of a Patient with Roberts Syndrome and a Rare ESCO2 Variant
}

\author{
Carla Bastos da Costa Almeida ${ }^{1}$ Amanda Thum Welter ${ }^{1}$ Gabriel Dotta Abech ${ }^{1}$ \\ Gabriela Rangel Brandão ${ }^{1}$ José Antônio Monteiro Flores ${ }^{2}$ Birgitt Schüle ${ }^{3}$ Uta Francke ${ }^{3}$ \\ Marilu Fiegenbaum ${ }^{4}$ Paulo Ricardo Gazzola Zen ${ }^{5,6}$ Rafael Fabiano Machado Rosa ${ }^{5,6}$
}

${ }^{1}$ Department of Medicine, Universidade Federal de Ciências da Saúde

Address for correspondence Rafael Fabiano Machado Rosa, MSc, de Porto Alegre, Rio Grande do Sul, Brazil

2 Pediatric Radiology Service, Hospital da Criança Santo Antônio/

Santa Casa de Misericórdia de Porto Alegre, Rio Grande do Sul, Brazil

${ }^{3}$ Department of Genetics and Pediatrics, Stanford University School PhD, Genética Clínica, Universidade Federal de Ciências da Saúde de Porto Alegre and Santa Casa de Misericórdia de Porto Alegre, Rua Sarmento Leite, 245/403, Porto Alegre, RS 90050-170, Brazil of Medicine, California, United States

${ }^{4}$ Department of Human Genetics, Universidade Federal de Ciências da Saúde de Porto Alegre, Rio Grande do Sul, Brazil

${ }^{5}$ Department of Clinical Genetics, Universidade Federal de Ciências da Saúde de Porto Alegre and Santa Casa de Misericórdia de Porto Alegre, Rio Grande do Sul, Brazil

${ }^{6}$ Department of Pathology, Universidade Federal de Ciências da

Saúde de Porto Alegre, Rio Grande do Sul, Brazil

J Pediatr Genet 2020;9:58-62.

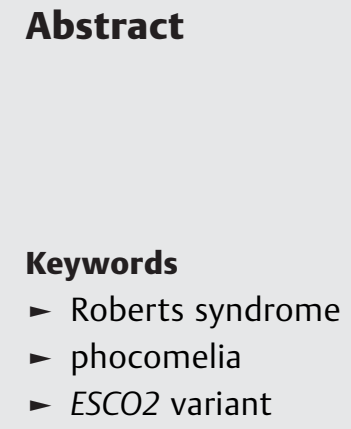

Abstract

\section{Introduction}

Roberts syndrome (RS) (OMIM \#268300) is a rare autosomal recessive genetic disorder, named after the report by John Roberts in $1919 .{ }^{1-4}$ There are earlier reports, however, in 1671 and 1737, which presented characteristics suggestive of RS. ${ }^{5,6}$ Herrmann et al also used, in 1969, the term "SC Phocomelia syndrome" or "thalidomide-like syndrome" (due to similarity of the clinical findings observed in patients exposed to thalidomide during pregnancy) in the description of two European families with clinical findings similar to RS..$^{7-10}$

Tomkins et al in 1979 described a cytogenetic alteration involving the centromere, which affected most of the chromosomes of RS patients, that was later called "premature separation of the centromere" by James German. ${ }^{11,12}$ Furthermore, it was found that individuals with RS exhibit a lack of cohesion involving the heterochromatic region of some chromosomes, those around the centromere and in the distal portion of the $\mathrm{Y}$ chromosome long arm (called heterochromatin repulsion [HR]). Currently, it is known that RS is caused by biallelic variants in the ESCO2 gene. ${ }^{3}$

Around 150 cases of RS have been described in the literature. $^{13}$ The condition is characterized by a broad range of clinical findings with variable expressivity, such as growth retardation (of pre- and postnatal onsets), craniofacial abnormalities (such as microcephaly and cleft palate), symmetrical limb reduction (tetraphocomelia), and varying degrees of intellectual disability. ${ }^{1,3,5,14}$ The prognosis of RS is generally received

March 31, 2019

accepted after revision

July 22, 2019

published online

September 3, 2019
Copyright (c) 2020 by Georg Thieme

Verlag KG, Stuttgart · New York
DOI https://doi.org/ 10.1055/s-0039-1696636. ISSN 2146-4596. 
poor, and survival usually does not exceed the neonatal period. ${ }^{15}$

Here, we report a patient with a rare ESCO2 variant.

\section{Case Report}

The patient was the first son of a young, healthy Caucasian couple, not known to be related to each other. There was no similar case in his family. The pregnancy was uneventful. A fetal ultrasound performed in the fifth month of gestation indicated a probable cleft lip and palate, and shortened long bones. The child was born at term by Cesarean section weighing $1,570 \mathrm{~g}$, measuring $34 \mathrm{~cm}$, with head circumference of $26 \mathrm{~cm}$, and Apgar scores of 9 and 10 in the first and fifth minutes, respectively.

Physical examination on the second day of life evidenced a wide anterior fontanelle (extended from the base of frontal bone to the superior occipital region); orbital hypertelorism; blepharophimosis; shallow orbits with exophthalmos; a central facial cleft involving the nose, lip, and palate with the presence of a nasal rudiment; and small, low-set, and posteriorly rotated ears. The extremities exhibited dramatic bilateral shortening of humerus and apparent bilateral absence of forearms with small hands, absence of the left thumb, and presence of an appendicular thumb on the right. He had bilateral shortening of thighs and legs, with restricted knee movement, syndactyly between fourth and fifth toes. Hypertrichosis was noted in the sacral regions and thighs, as well as a hypertonic posture with neck extension. He had normal male genitalia (-Fig. 1).

Radiographic evaluation revealed the presence of marked deformity of the skull with reduction of its anteroposterior diameter; eleven ribs; shortened and deformed humerus; absent radius; right hand presenting two metacarpal bones with normal appearance, two fused metacarpals and four fingers with phalanges; left hand with three metacarpal bones and four fingers with phalanges; femurs were bent, and tibia and fíbula were absent bilaterally ( - Fig. 2). Brain and abdominal ultrasound examinations were normal. Cardiological evaluation showed interventricular communication and tricuspid regurgitation. The patient was deteriorated and died at 20 days of age.

High-resolution GTG-banded karyotype made with peripheral lymphocytes showed a male chromosomal constitution $(46, \mathrm{XY})$. There was also lack of centromeric constriction in some chromosomes, premature centromere separation (PCS) in others, and regions of HR (most evident on chromosomes 1, 9, 16, and Y). The total number of scored cells was 32. The percentage displaying PCS/HR phenotype was $100 \%$, and the number of chromosomes with PCS/HR per cell was 8 (-Fig. 3). Molecular analysis from DNA extracted from peripheral blood of the patient through sequencing of all 10 coding exons and splice junctions of the ESCO2 gene using BigDye Terminator chemistry and an ABI 3100 sequencer (Applied Biosystems, Foster City, California, United States) revealed a deletion of $4 \mathrm{bp}$ involving exon 4 in homozygosity (NM_001017420.2:c.875_878delACAG, rs80359856) (-Fig. 4). Both parents were heterozygotes for this variant. This variant causes a frameshift and premature stop codon. Because of nonsense-mediated decay of the mutant mRNA, the predicted truncated protein $\mathrm{p}$. (Asp292Glufs) is unlikely to be produced in significant quantities. A subsequent pregnancy of this couple was monitored, and the fetus was found to be homozygous for the normal allele ( - Fig. 4). No other family members were tested for this variant.

\section{Discussion}

In our literature review in MEDLINE, we found only one Brazilian study involving individuals with RS. Barbosa et al evaluated the replication patterns of homologous alphoid
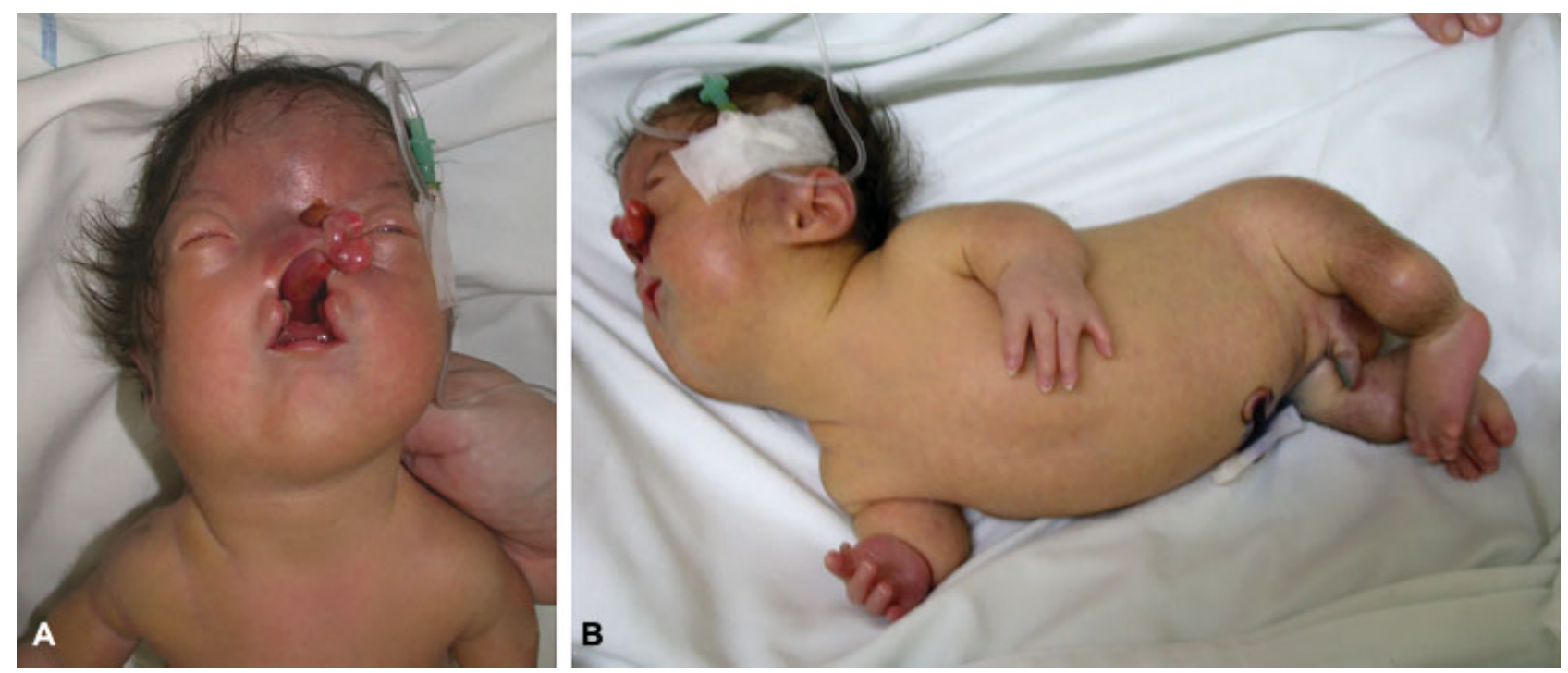

Fig. 1 Patient at 2 days of age. Note orbital hypertelorism and facial cleft involving the nose, lip, and palate with the presence of a nasal rudiment (A); small, low-set, and posteriorly rotated ears; drastic shortening of the arm and forearm along with small hands with absence of thumb on the left side and presence of an appendicular thumb on the right, and remarkable shortening of thighs and legs (B). 


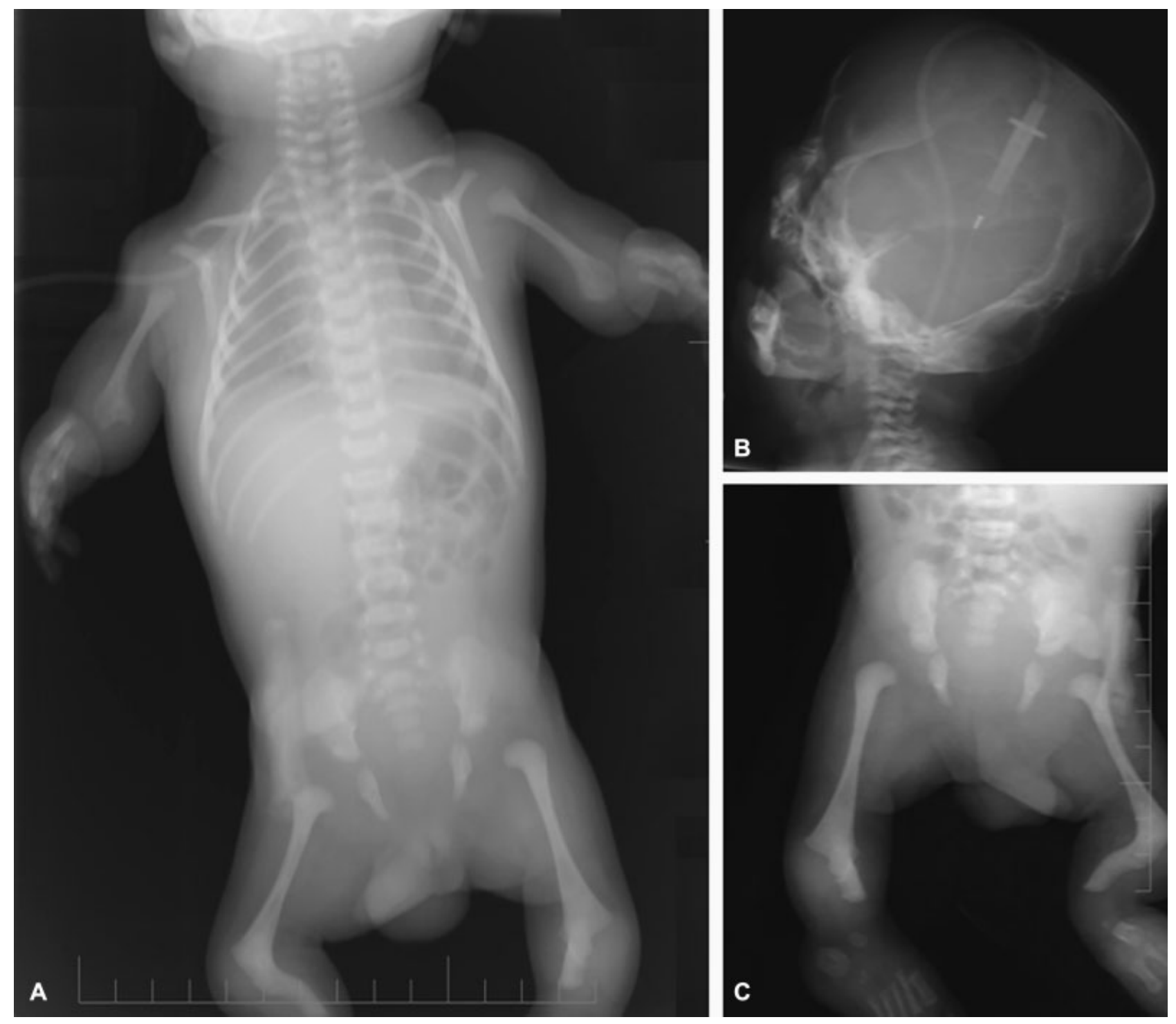

Fig. 2 Radiographic evaluation showing 11 ribs and shortened and deformed humerus (A); marked deformity of the skull with reduction of its anteroposterior diameter (B), and femur bending, with absence of tibia and fibula (C).

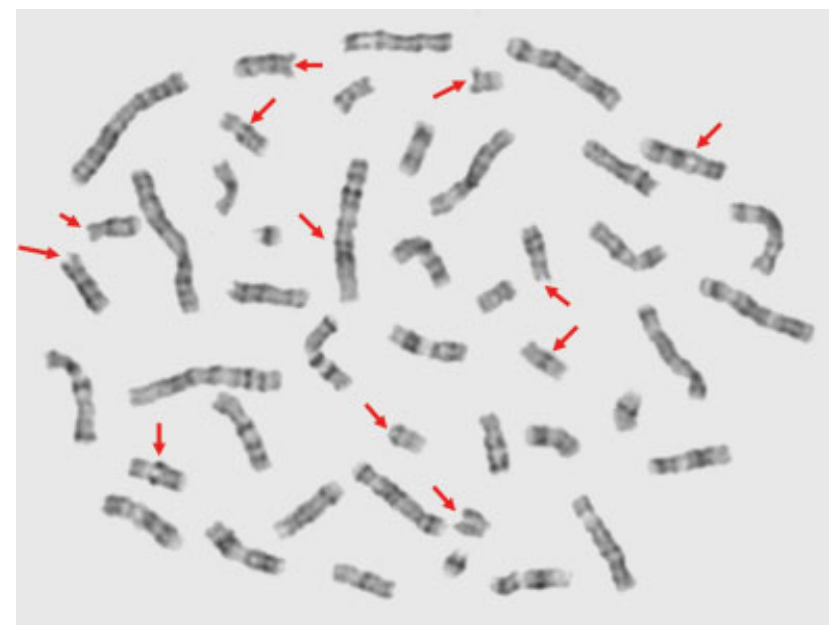

Fig. 3 High-resolution GTG-banded karyotype revealing lack of centromeric constriction in some chromosomes, premature centromere separation in others, and repulsion of the heterochromatin regions (see red arrows). centromeric DNA of chromosomes 9, 11, 16, and 17 using fluorescence in situ hybridization analysis. They analyzed three patients. However, there was no description of their clinical features. ${ }^{15}$ Searching other databases from Latin America, we verified the presence of three studies, one from Brazil and the other two from Cuba and Venezuela. This Brazilian study by Gollop et al claimed that it was the first case of RS described in Brazil. ${ }^{16}$ The diagnosis was made by fetal ultrasound during pregnancy and was based on the findings of tetraphocomelia, marked retromicrognathia without lip-palatine fissures or premaxillary protrusions. These findings were confirmed later by autopsy. In both reports, there was no description of additional genetic evaluations, as the gene for RS was discovered only years later. Therefore, our case represents the first molecularly confirmed case of RS in Brazil.

Our patient also presented abnormalities identified through karyotype analysis that consisted of lack of centromeric constriction in some chromosomes, PCS in others, and 

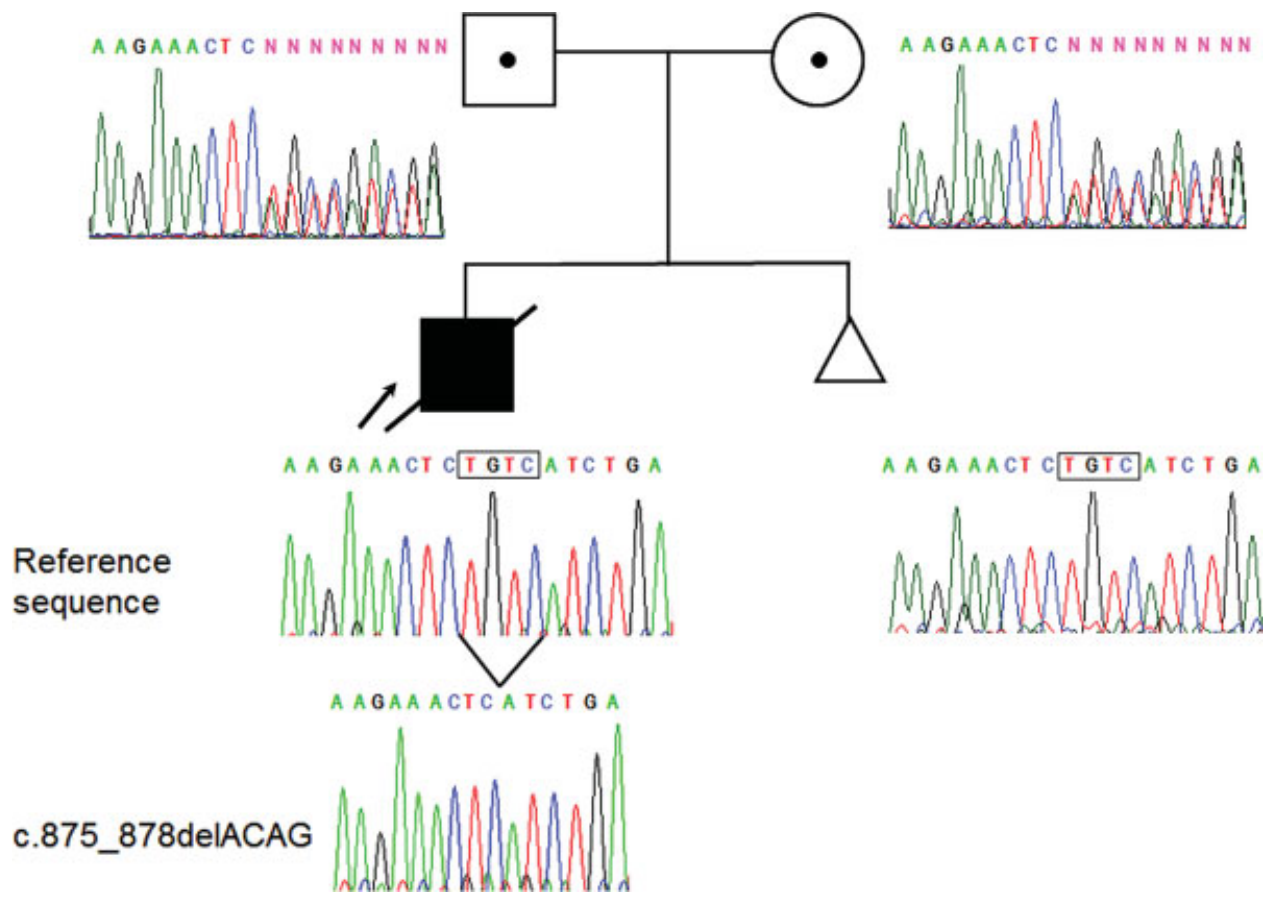

Fig. 4 Family tree with DNA sequence tracings of the reverse strand. Both parents are heterozygous for the deletion. The proband is homozygous for the c.875_878delACAG deletion. A subsequent pregnancy was monitored prenatally, and the fetus was homozygous for the normal allele (identical to the reference sequence shown).

repulsion of heterochromatin regions. These are considered pathognomonic findings for the diagnosis of RS. ${ }^{2}$ These cytogenetic findings are described in $79.1 \%$ of the patients with RS. ${ }^{3}$ Studies have suggested that the lack of fusion of heterochromatin in RS would be caused by the variant in ESCO2. This ensures that the cohesin ring around the sister chromatids establishes cohesion during the $S$ phase of replication along with ESCO1.,2,10,16-19

As for prenatal diagnosis, we observed, in our case, the ultrasonographic description in the fifth month of gestation of a probable cleft lip and palate. Moreover, shortening of the long bones was evident. Literature reports of prenatal diagnosis of RS most frequently describe reduced fetal movements, restriction of fetal growth, and shortening of the long bones. $2,5,9,12,18,20-22$ In the case of prenatal diagnosis reported by Gollop et al, those findings were observed during a standard ultrasound at 21 weeks of gestation. ${ }^{23}$

The clinical manifestations observed in our patient did not differ from the majority of RS cases described in the literature with RS (- Table 1). ${ }^{22}$ This suggests that RS is not clinically variable. Few patients with RS also survive for more than 1 month, which is compatible with the clinical evolution observed in our case. ${ }^{22}$

The ESCO2 variant identified in our patient was described once before by Gordillo et al in a French family, although no clinical data were provided. This variant is not reported in the Genome Aggregation Database (gnomAD) ${ }^{24}$ In ClinVar, it is described once. ${ }^{25}$ In the Exome Aggregation Consortium (ExAC), the variant allele was also reported only once, in the Asian population (minor allele frequency: $8.299 \mathrm{e}-06$ ). ${ }^{26}$ According to the College of Medical Genetics and Genomics and the Association for Molecular Pathology Standards and
Table 1 Clinical features of Roberts syndrome described in the literature in comparison with our patient (based on Van Den Berg and Francke ${ }^{22}$ )

\begin{tabular}{|l|l|l|}
\hline Clinical features & $\begin{array}{l}\text { Literature } \\
\%\end{array}$ & Our patient \\
\hline Consanguinity & 40.8 & - \\
\hline Sex & $54 \mathrm{M} / 39 \mathrm{~F} / 7 \mathrm{U}$ & $\mathrm{M}$ \\
\hline Neurological & \multicolumn{2}{|l|}{} \\
\hline Mental retardation & 79.4 & $\mathrm{X}$ \\
\hline Growth retardation & 97.5 & + \\
\hline Craniofacial anomalies & \multicolumn{2}{|l|}{} \\
\hline Microcephaly & 80.4 & \\
\hline Hemangioma & 29 & + \\
\hline $\begin{array}{l}\text { Exophthalmia/ } \\
\text { prominent eyes }\end{array}$ & 69.4 & + \\
\hline Hypertelorism & 86.7 & \\
\hline Cloudy cornea & 68.1 & \\
\hline $\begin{array}{l}\text { Hypoplastic } \\
\text { nostrils }\end{array}$ & 32 & + \\
\hline Cleft palate & 60.6 & + \\
\hline Cleft lip & 49 & \\
\hline $\begin{array}{l}\text { Highly arched } \\
\text { palate }\end{array}$ & 16 & (Continued) \\
\hline Prominent maxilla & 30 & \\
\hline Micrognathia & 13 & 75.9 \\
\hline Abnormal ears & & \\
\hline \multicolumn{2}{|l|}{} \\
\hline
\end{tabular}


Table 1 (Continued)

\begin{tabular}{|c|c|c|}
\hline Clinical features & $\begin{array}{l}\text { Literature } \\
\%\end{array}$ & Our patient \\
\hline \multicolumn{3}{|l|}{ Limb anomalies } \\
\hline Phocomelia & 100 & + \\
\hline Ulnar aplasia & 96.5 & + \\
\hline Radial aplasia & 97.8 & + \\
\hline Femoral aplasia & 64.9 & \\
\hline Tibial aplasia & 74.1 & + \\
\hline Fibular aplasia & 80.5 & + \\
\hline Leg bone synostosis & 7 & \\
\hline Syndactyly & 42.5 & + \\
\hline Flexion contractures & 51 & + \\
\hline \multicolumn{3}{|l|}{ Other anomalies } \\
\hline Heart defects & 13 & + \\
\hline Kidney anomalies & 50 & \\
\hline Enlarged phallus & 53.2 & + \\
\hline Cryptorchidism & 51.2 & \\
\hline
\end{tabular}

Abbreviations: F, female; $M$, male; $U$, undetermined sex; $X$, unknown.

Guidelines, the variant was classified as pathogenic based on the following criteria: causing a frameshift of extremely low frequency in ExAC and not described in the 1000 Genomes project; the protein length changes (normal protein: 602 aa; mutated protein: $339 \mathrm{aa}$ ); and other support computational evidences that the variant is deleterious, and it has a relationship with the observed phenotype. ${ }^{27}$

Thus, the variant found in our patient is considered a rare ESCO2 variant, whose phenotype was not previously described. This variant causes loss of function of ESCO2, and the clinical findings and clinical course of our patient were typical for RS cases. This represents the first Brazilian report of a patient with RS describing his clinical features.

Conflict of Interest

None declared.

\section{References}

1 McDaniel LD, Prueitt R, Probst LC, et al. Novel assay for Roberts syndrome assigns variable phenotypes to one complementation group. Am J Med Genet 2000;93(03):223-229

2 Schüle B, Oviedo A, Johnston K, Pai S, Francke U. Inactivating mutations in ESCO2 cause SC phocomelia and Roberts syndrome: no phenotype-genotype correlation. Am J Hum Genet 2005;77 (06):1117-1128

3 Vega $\mathrm{H}$, Waisfisz Q Gordillo M, et al. Roberts syndrome is caused by mutations in ESCO2, a human homolog of yeast ECO1 that is essential for the establishment of sister chromatid cohesion. Nat Genet 2005;37(05):468-470

4 Roberts JB. A child with double cleft lip and palate, protrusion of the intermaxillary portion of the upper jaw and imperfect development of the bones of the four extremities. Ann Surg 1919;70:252-254

5 Bates AW. A case of Roberts syndrome described in 1737. J Med Genet 2001;38(08):565-567
6 Oostra RJ, Baljet B, Dijkstra PF, Hennekam RC. Congenital anomalies in the teratological collection of Museum Vrolik in Amsterdam, The Netherlands. I: syndromes with multiple congenital anomalies. Am J Med Genet 1998;77(02):100-115

7 Herrmann J, Feingold M, Tuffli GA, Opitz JM. A familial dysmorphogenetic syndrome of limb deformities, characteristic facial appearance and associated anomalies: the pseudothalidomide or SC-syndrome. Birth Defects Orig Art Ser 1969;5:81-89

8 Herrmann J, Opitz JM. The SC phocomelia and the Roberts syndrome: nosologic aspects. Eur J Pediatr 1977;125(02):117-134

9 Al Kaissi A, Csepan R, Klaushofer K, Grill F. Femoral-tibial-synostosis in a child with Roberts syndrome (pseudothalidomide): a case report. Cases J 2008;1(01):109

10 Sánchez-Segura M, Marsán-Suárez V, Macías-Abraham C, et al. Roberts syndrome associated with immunodeficiency. Rev Cubana Hematol Inmunol Hemoter 2012;28(02):185-191

11 Tomkins D, Hunter A, Roberts M. Cytogenetic findings in RobertsSC phocomelia syndrome(s). Am J Med Genet 1979;4(01):17-26

12 Schulz S, Gerloff C, Ledig S, et al. Prenatal diagnosis of Roberts syndrome and detection of an ESCO2 frameshift mutation in a Pakistani family. Prenat Diagn 2008;28(01):42-45

13 Zhou J, Yang X, Jin X, Jia Z, Lu H, Qi Z. Long-term survival after corrective surgeries in two patients with severe deformities due to Roberts syndrome: a case report and review of the literature. Exp Ther Med 2018;15(02):1702-1711

14 Gordillo M, Vega H, Trainer AH, et al. The molecular mechanism underlying Roberts syndrome involves loss of ESCO2 acetyltransferase activity. Hum Mol Genet 2008;17(14):2172-2180

15 Barbosa AC, Otto PA, Vianna-Morgante AM. Replication timing of homologous alpha-satellite DNA in Roberts syndrome. Chromosome Res 2000;8(07):645-650

16 German J. Roberts' syndrome. I. cytological evidence for a disturbance in chromatid pairing. Clin Genet 1979;16(06):441-447

17 Louie E, German J. Roberts's syndrome. II. Aberrant Y-chromosome behavior. Clin Genet 1981;19(01):71-74

18 Vega $\mathrm{H}$, Trainer $\mathrm{AH}$, Gordillo $\mathrm{M}$, et al. Phenotypic variability in 49 cases of ESCO2 mutations, including novel missense and codon deletion in the acetyltransferase domain, correlates with ESCO2 expression and establishes the clinical criteria for Roberts syndrome. J Med Genet 2010;47(01):30-37

19 Goh ES-Y, Li C, Horsburgh S, Kasai Y, Kolomietz E, Morel CF. The Roberts syndrome/SC phocomelia spectrum-a case report of an adult with review of the literature. Am J Med Genet A 2010;152A (02):472-478

20 Petrinelli P, Antonelli A, Marcucci L, Dallapiccola B. Premature centromere splitting in a presumptive mild form of Roberts syndrome. Hum Genet 1984;66(01):96-99

21 Parry DM, Mulvihill JJ, Tsai SE, Kaiser-Kupfer MI, Cowan JM. SC phocomelia syndrome, premature centromere separation, and congenital cranial nerve paralysis in two sisters, one with malignant melanoma. Am J Med Genet 1986;24(04):653-672

22 Van Den Berg DJ, Francke U. Roberts syndrome: a review of 100 cases and a new rating system for severity. Am J Med Genet 1993; 47(07):1104-1123

23 Gollop TR, Eigier A, Hauschild D, Guidugli J, Moron AF. Prenatal ultrasound diagnosis of Roberts syndrome at 21 weeks. Braz J Genet 1990;13(03):607-612

24 The Genome Aggregation Database (gnomAD). Retrieved from https://gnomad.broadinstitute.org/. Accessed April 25, 2019

25 ClinVar. Retrieved from https://www.ncbi.nlm.nih.gov/clinvar/. Accessed September 4, 2018

26 ExAC Browser (Beta)|Exome Aggregation Consortium. Retrieved from http://exac.broadinstitute.org/. Accessed September 4, 2018

27 Richards S, Aziz N, Bale S, et al; ACMG Laboratory Quality Assurance Committee. Standards and guidelines for the interpretation of sequence variants: a joint consensus recommendation of the American College of Medical Genetics and Genomics and the Association for Molecular Pathology. Genet Med 2015;17(05):405-424 